\title{
Percepción del funcionamiento familiar por estudiantes de nivel medio superior de una universidad pública
}

\section{Perception of family functioning by high school students at a public university}

DOI: $10.46932 / \mathrm{sfjdv} 2 \mathrm{n} 2-043$

Received in: january 1st, 2020

Accepted in: March 30th, 2020

\section{Guillermina García Madrid}

DCF

Profesor Investigador recién Jubilado de la Facultad de Enfermería de la Benemérita Universidad Autónoma de Puebla. guillergm25@ hotmail.com

\section{Maricarmen Moreno Tochihuitl \\ DCE.}

Profesor Investigadores de la Facultad de Enfermería de la Benemérita Universidad Autónoma de Puebla

\section{Carmen Cruz Rivera}

MCE.

Profesor Investigadores de la Facultad de Enfermería de la Benemérita Universidad Autónoma de Puebla

\section{Jorge Antonio Ramos Vázquez}

ME.

Profesor Investigadores de la Facultad de Enfermería de la Benemérita Universidad Autónoma de Puebla

\section{María Laura Méndez Ovando}

Lic. Psic.

Profesor Investigadores de la Facultad de Enfermería de la Benemérita Universidad Autónoma de Puebla

\section{Irma Vera Sánchez \\ Dra.}

Profesor Investigadores de la Facultad de Enfermería de la Benemérita Universidad Autónoma de Puebla MCE.

\section{Verónica Pérez Badillo}

Profesor Investigadores de la Facultad de Enfermería de la Benemérita Universidad Autónoma de Puebla

\section{José Gulmaro Pérez Juárez MCE.}

Profesor de la Escuela de Enfermería de la Universidad del Valle de Puebla. 


\title{
RESUMEN
}

En el marco del Cuidado de la Salud Familiar patrocinado por el Consejo Internacional de Enfermería (CIE, 2002) y respaldado en México por el Programa "Escuela Saludable" de la Secretaría de Salud (S. S., 2010), el estudio del funcionamiento familiar, es un indicador elemental a realizar en las escuelas a los estudiantes, debido a su relación con el rendimiento escolar. Por ello, el objetivo del trabajo fue identificar cómo perciben el funcionamiento de sus familias los estudiantes de nivel preparatoria de una universidad pública de la ciudad de Puebla, México. Se trabajó con 216 estudiantes seleccionados aleatoriamente a quienes se les aplicó la Escala de Efectividad en el Funcionamiento Familiar de la Dra. Ma. Louise Friedemann. La edad de los estudiantes osciló entre los 13 y 18 años, las familias se caracterizaron porque el $71.4 \%$ son de tipo nuclear, el $40.8 \%$ tiene un promedio de 5 a 6 integrantes, el $60.6 \%$ es de una comunidad rural, el $80.6 \%$ es de un nivel socioeconómico medio y el $82 \%$ practica la religión católica. En relación a la percepción del funcionamiento familiar el $44.18 \%$ de estudiantes la percibe con un funcionamiento familiar alto, el $18.44 \%$ con un funcionamiento familiar medio y el $37.38 \%$ con un funcionamiento familiar bajo. La dimensión mantenimiento del sistema relacionada con las costumbres, creencias, hábitos, rutinas, reglas, normas y patrones de crianza tuvo la media más alta $52.29(D E=5.66)$ y la dimensión coherencia que engloba las relaciones familiares, la expresión de lazos de afecto, amor cariño, comunicación, apoyo y convivencia; factores que le dan un sentido de unidad, pertenencia y compromiso a la familia la media más baja $16.39(D E=5.27)$. Se concluye que la percepción del funcionamiento familiar depende de alguna manera de la etapa de vida de las personas encuestadas.

Palabras clave: funcionamiento familiar, estudiantes, nivel medio superior.

\begin{abstract}
Within the framework of Family Health Care sponsored by the International Council of Nursing (ICN, 2002) and supported in Mexico by the "Healthy School" Program of the Ministry of Health (S. S., 2010), the study of family functioning is an elementary indicator to be performed in schools to students, due to its relationship with school performance. Therefore, the objective of this study was to identify how high school students of a public university in the city of Puebla, Mexico, perceive the functioning of their families. We worked with 216 randomly selected students who were administered Dr. Ma. Louise Friedemann's Family Functioning Effectiveness Scale. The age of the students ranged between 13 and 18 years, the families were characterized by the fact that $71.4 \%$ are nuclear, $40.8 \%$ have an average of 5 to 6 members, $60.6 \%$ are from a rural community, $80.6 \%$ have a medium socioeconomic level and $82 \%$ practice the Catholic religion. In relation to the perception of family functioning, $44.18 \%$ of the students perceive it as high family functioning, $18.44 \%$ as medium family functioning and $37.38 \%$ as low family functioning. The dimension maintenance of the system related to customs, beliefs, habits, routines, rules, norms and patterns of upbringing had the highest mean $52.29(\mathrm{SD}=5.66)$ and the dimension coherence that encompasses family relationships, the expression of bonds of affection, love, communication, support and coexistence; factors that give a sense of unity, belonging and commitment to the family had the lowest mean 16.39 ( $\mathrm{SD}=5.27$ ). It is concluded that the perception of family functioning depends in some way on the life stage of the respondents.
\end{abstract}

Key words: family functioning, students, high school level.

\section{INTRODUCCIÓN}

En el marco del Cuidado de la Salud Familiar patrocinado por el Consejo Internacional de Enfermeras (CIE, 2002) y respaldado en México por el Programa "Escuela Saludable" de la Secretaría de 
Salud (S. S., 2010). El estudio del funcionamiento familiar, es un indicador elemental a realizar en las escuelas a los estudiantes, debido a su relación con el rendimiento escolar. Este es considerado por la Dra. Ma. Luisa Friedemann (1995) autora del Marco de Organización Sistémica (MOS) como la capacidad que tiene la familia de mantener la congruencia o armonía ante las situaciones estresantes generadoras de ansiedad y los cambios o retos que le plantea la vida en el desarrollo de su ciclo vital familiar. Aunque de acuerdo a este marco teórico la salud familiar nunca es del todo alcanzada, sus integrantes generalmente hacen todo lo que pueden por conseguirla; para ello implementan estrategias de conducta en las dimensiones de su proceso de vida denominadas mantenimiento del sistema, cambio del sistema, individuación y coherencia y lograr la estabilidad, el control, crecimiento y espiritualidad como metas de la familia.

La dimensión mantenimiento del sistema, comprende todas aquellas acciones que realiza la familia para protegerse de los cambios amenazantes generadores de ansiedad y mantenerse como sistema: creencias, hábitos y costumbres, reglas y normas, patrones de crianza etc. La dimensión cambio del sistema, muestra las respuestas conscientes de esta al cambio dentro del sistema familiar, implica la toma de decisiones, la adopción de la nueva información, la modificación o reemplazo de viejos valores, actitudes y conductas en la familia. La dimensión individuación, consiste en la incorporación de conductas de crecimiento y desarrollo físico e intelectual individual, que les enseña a los integrantes de la familia acerca de sí mismos y de otros y los llevan a tener un propósito de vida. Y la dimensión coherencia engloba las relaciones armónicas y la expresión de lazos de afecto, amor, cariño, comunicación, apoyo y convivencia, comportamientos que hacen que se mantenga la familia y que le dan un sentido de unidad, pertenencia y compromiso familiar.

Por otro lado el logro de cada una de las metas de la familia engloba comportamientos de dos dimensiones de su proceso de vida. La meta control, integra comportamientos relacionados a las dimensiones mantenimiento del sistema y cambio del sistema. La meta crecimiento, incluye acciones de las dimensiones cambio del sistema e individuación. La meta espiritualidad, incorpora estrategias de las dimensiones de individuación y coherencia. Y finalmente la meta estabilidad, suma comportamientos de las dimensiones coherencia y mantenimiento del sistema En la teoría los procesos de vida del sistema familiar y las metas de la familia interactúan entre sí para mantener un equilibrio entre estas y lograr que la familia se conserve saludable (Ver figura 1).

Figura 1. Marco de Organización Sistémica: un Enfoque Conceptual para Familias y Enfermería Friedemann (1995). 


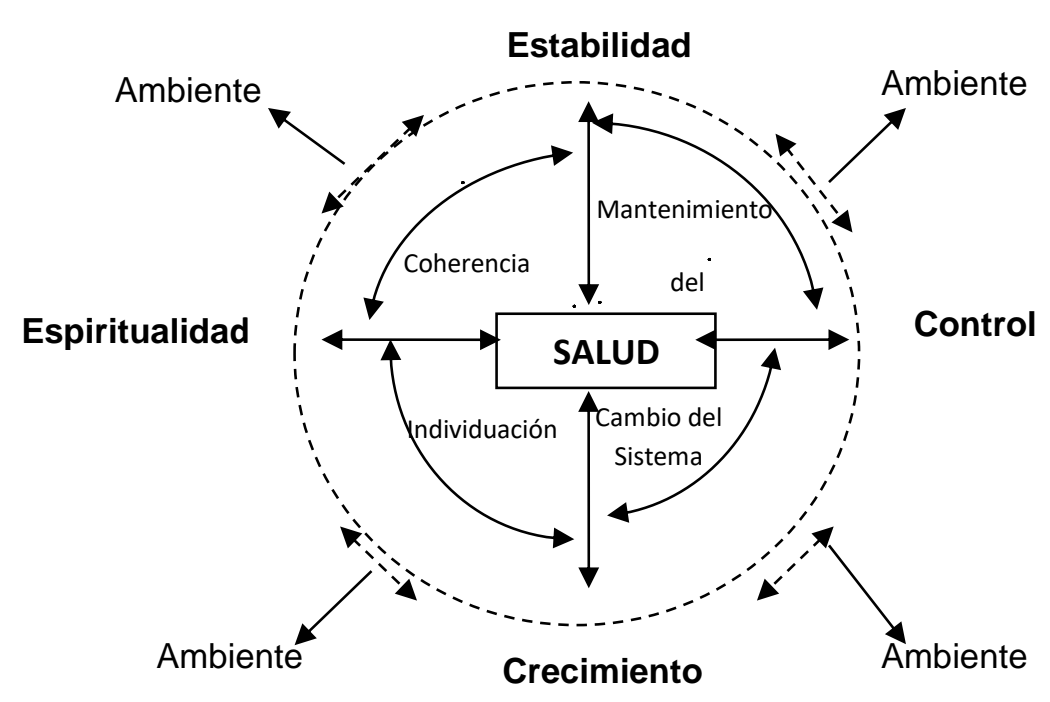

Según las Naciones Unidas (1988, citado en Membrillo, Fernández, Quiroz y Rodríguez 2008), el cumplimiento de las funciones de la familia son básicas para el desarrollo saludable de sus miembros y el funcionamiento de la sociedad, entre estas se especifican las siguientes: satisfacer sus necesidades básicas, proporcionarles cuidado, amor, cariño y afecto, permitirles la expresión de su sexualidad, socializar a los hijos mediante la comunicación y un medio de desarrollo intelectual, emocional e interpersonal que favorezca su bienestar psicosocial como es la escuela. Es por ello que para la Dra. Friedemann (1995) la familia es un sistema social abierto que se relaciona con todos los demás sistemas de la sociedad humana.

Cada familia tiene un estilo propio de cumplir con sus funciones, pero cuando estas no son satisfechas la familia puede disfuncionar. Una familia disfuncional o con un funcionamiento inadecuado, es un factor de riesgo, para la salud de los individuos, al favorecer en ellos la aparición de síntomas y enfermedades físicas y emocionales. En cambio una familia funcional o con un funcionamiento adecuado, promueve el desarrollo integral de sus miembros, al lograr el mantenimiento de estados de salud favorables en éstos (Herrera, 1997).

Como la salud familiar y la salud individual, son un proceso de doble vía y su influencia es recíproca, el funcionamiento familiar infiere de alguna manera en la salud física y emocional de sus integrantes y por lo tanto en su crecimiento y desarrollo personal. En la medida en que el funcionamiento de sus familias sea eficaz estos tendrán un mejor o peor desempeño escolar. De ahí que se relacione con el rendimiento y desempeño escolar en los estudiantes en todos sus niveles académicos, así lo demuestran estudios como el de Torres y Rodríguez (2006) y Santana (2008) quienes al estudiar el rendimiento escolar en relación al funcionamiento familiar afirman que el desempeño escolar depende del contexto familiar en el que se desarrolle el estudiante y de que uno de los factores que consideraban obstaculizaba su aprovechamiento eran los problemas familiares y personales, por lo que es importante conocer la percepción que los jóvenes tienen acerca de la relación positiva o negativa con su familia, en relación al 
apoyo que esta les presta, del cumplimiento de sus necesidades, tareas y expectativas, de la comunicación y preocupación que muestran con ellos y de las normas y reglas establecidas. Por su parte, Paz y Aymat (2007) señalan que los estudiantes cuyas familias son disfuncionales tienen una frecuencia significativamente mayor de problemas en el desempeño escolar.

Por otro lado González (2004) al estudiar la funcionalidad familiar en adolescentes de nivel medio y superior encontró que un gran porcentaje percibe a su familia con un funcionamiento bajo y que existe una asociación negativa de la edad con el funcionamiento familiar $(r=.-35, p=.01)$; Criado et al (2009) por el contrario informaron que en su estudio obtuvieron que un porcentaje por arriba de la media de estudiantes reportaron a sus familias con un funcionamiento familiar bajo y que la dimensión mantenimiento del sistema fue la más alta y la dimensión cambio del sistema la mas baja; Morales y Rubiano, (2009) analizaron el funcionamiento familiar en familias con adolescentes, encontraron que el $2 \%$ tuvo un funcionamiento familiar adecuado, el $32.7 \%$ funcionamiento familiar moderado y el $65.3 \%$ un funcionamiento familiar bajo. El 74\% tuvo un alto nivel en la dimensión mantenimiento del sistema, el $6 \%$ un nivel bajo en la dimensión coherencia y un 70\% un nivel alto en el logro de la meta estabilidad. Guadarrama, Márquez, Veytia, López y León (2011) al estudiar el funcionamiento familiar en estudiantes universitarios de seis diferentes licenciaturas de una universidad pública también reportaron que un gran número de estudiantes percibe a su familia como disfuncional, ya que no tienen con ella una comunicación adecuada. Sin embargo, en este mismo estudio los estudiantes también resaltan algunos aspectos positivos de su familia como es el sentirse parte de ella y la ayuda que reciben ante la enfermedad, por lo que los autores señalan que los estudiantes son personas que necesitan desarrollar no sólo sus capacidades académicas, sino también sus capacidades personales y familiares para desarrollarse de manera exitosa profesionalmente. Es por ello que la escuela tiene la función de que los estudiantes adquieran competencias cognitivas, procedimentales y actitudinales que los transformen en individuos socialmente productivos.

En la revisión de la literatura se observó que el fenómeno de estudio ha sido abordado por disciplinas como la psicología, la educación y la medicina, sin embargo, es poca la evidencia científica generada por enfermería, disciplina cuya esencia es el cuidado de la salud humana y de la salud familiar. Es por ello, el interés de conocer como perciben el funcionamiento de sus familias los estudiantes de nivel medio superior de una universidad pública con base al Marco Teórico de Organización Sistémica: Un enfoque conceptual orientado al cuidado de la salud familiar desde la disciplina de enfermería, de tal manera que los resultados permitan implementar intervenciones de enfermería que fortalezcan a los estudiantes en su desempeño escolar, el programa de escuela saludable y la salud familiar. 


\section{METODOLOGÍA}

El estudio es de tipo descriptivo, correlacional, transversal, realizado en 216 alumnos de nivel preparatoria de una universidad pública de la ciudad de Puebla, México, seleccionados mediante un muestreo probabilístico aleatorio simple. Para la recolección de la información se utilizó una cédula de factores personales y familiares y la Escala de Efectividad en el Funcionamiento Familiar (E-EFF24), versión latina, elaborada por Chávez, Friedemann y Alcorta en el año 2000, readaptada por García en el año 2005, las cuales fueron aplicadas previo permiso de las autoridades de la institución educativa en los salones de clase.

En el desarrollo del estudio se preservó el respeto a la dignidad y bienestar de los estudiantes, se les informó de manera clara, sencilla y concreta el motivo de la investigación y se les pidió su autorización por escrito a través del consentimiento informado. A los que eran menores de edad se les solicitó la autorización de los padres como lo establece el Reglamento de la Ley General de Salud en Materia de Investigación (Diario Oficial de la Federación 1984:2012). De igual manera se evitó emitir juicios personales respecto al funcionamiento familiar, al término del estudio se agradeció a los participantes su colaboración y todos los instrumentos fueron resguardados en un lugar seguro en donde solo la investigadora tenía acceso a ellos. La captura y análisis de los datos se hizo en el paquete estadístico SPSS (Statiscal Package for the Social Sciencies) versión 19.0. Se utilizaron estadísticos descriptivos y paramétricos de acuerdo a la curva de normalidad de los datos previa conversión en índices.

\section{RESULTADOS}

El rango de edad de los estudiantes se encontró entre los 13 y 18 años, el 50\% fueron mujeres y el $50 \%$ hombres, el $83.5 \%$ son solteros, el $3.4 \%$ están casados, un $10.2 \%$ son divorciados y un $2.9 \%$ viven en unión libre. Las familias de los estudiantes se caracterizan porque el $71.4 \%$ son de tipo nuclear, el $40.8 \%$ tiene un promedio de cinco o seis integrantes, el $80.6 \%$ es de nivel socioeconómico medio y el $82 \%$ practica la religión católica. En relación a la variable de estudio que es la percepción del funcionamiento familiar, el $44.18 \%$ la percibe con un funcionamiento familiar alto, el $18.44 \%$ con un funcionamiento familiar medio y el $37.38 \%$ con un funcionamiento familiar bajo como lo muestra la siguiente tabla.

Percepción del funcionamiento familiar de estudiantes de nivel medio superior de una universidad pública de la ciudad de Puebla, México.

\begin{tabular}{cllc}
\hline Variable & \multicolumn{1}{c}{ Grado } & $f$ & $\%$ \\
\hline \multirow{3}{*}{ Funcionamiento familiar } & Funcionamiento familiar alto & 96 & 44.18 \\
& Funcionamiento familiar medio & 39 & 18.44 \\
& Funcionamiento familiar bajo & 81 & 37.38 \\
\hline
\end{tabular}

$n=216$ estudiantes

Fuente: EE-FF24. Puebla, 2014. 
De las dimensiones de vida del sistema familiar la dimensión mantenimiento del sistema relacionada con las creencias, hábitos, costumbres, reglas, normas y patrones de crianza tuvo la media más alta $52.29(D E=5.66)$ y la dimensión coherencia que engloba las relaciones armónicas, la expresión de lazos de afecto, amor, cariño, comunicación, apoyo y convivencia que le dan un sentido de unidad, pertenencia y compromiso a la familia la más baja $16.39 \quad(D E=5.27)$ como se observa en la siguiente tabla.

Estadística descriptiva de las dimensiones de vida del funcionamiento familiar percibido por estudiantes de nivel medio superior de una universidad pública de la ciudad de Puebla, México.

\begin{tabular}{lcccc}
\multicolumn{1}{c}{ Variables } & Valor Mínimo & Valor Máximo & Media & $D E$ \\
\hline Mantenimiento del sistema & 33.33 & 62.50 & 52.29 & 5.66 \\
Cambio del sistema & 14.58 & 37.50 & 28.44 & 4.46 \\
Individuación & 16.67 & 33,33 & 26.93 & 3.62 \\
Coherencia & 6.25 & 81.25 & 16.39 & 5.27 \\
\hline
\end{tabular}

$n=216$ estudiantes

Fuente: EE-FF24. Puebla, 2014.

En cuanto al logro de las metas, la figura 2 muestra que la meta control tuvo la media más alta 80.73 y la meta espiritualidad la más baja 43.32, Sin embargo, no se encontró relación alguna con las variables edad, sexo y grado académico.

Figura 2. Logro de metas de la familia percibidas por estudiantes de nivel medio superior de una universidad pública de la ciudad de Puebla, México.

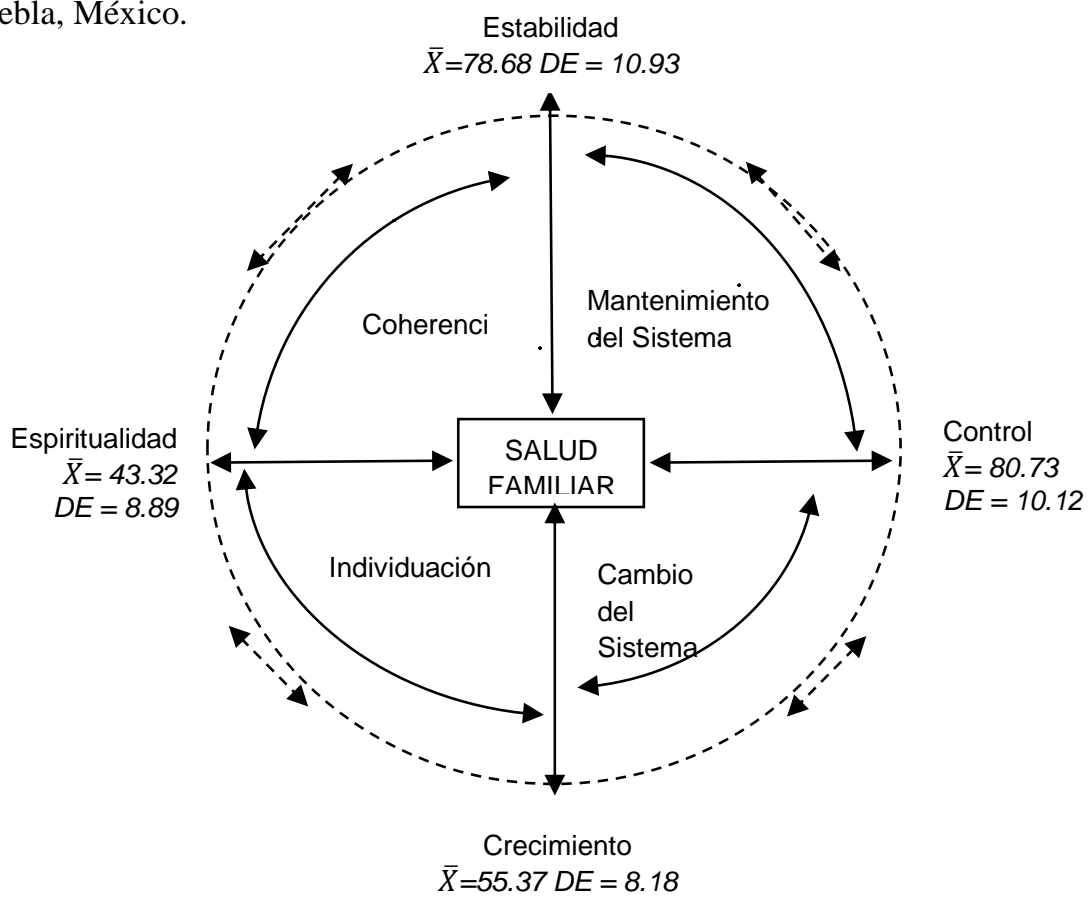

Fuente: EE-FF24. Puebla, 2014, $n=216$ estudiantes

\section{DISCUSIÓN}


La familia, tiene la tarea de proveer de bienestar a sus miembros durante el ciclo vital humano. Para ello se plantea metas y estrategias que aminoren la ansiedad y mantengan la congruencia del sistema familiar. En la medida en que el funcionamiento de las familias sea eficaz estos tendrán un mejor o peor crecimiento y desarrollo personal.

Lo antes descrito fue el fundamento teórico para el presente estudio. Los resultados obtenidos, permitieron dar respuesta al objetivo planteado. En ellos se encontró casi un equilibrio entre los rubros de corte del funcionamiento familiar (alto, medio y bajo). Datos similares a los reportados por González (2004) y Guadarrama, Márquez, Veytia, López y León (2011) quienes reportaron que un gran número de estudiantes percibe a su familia como disfuncional o con un funcionamiento familiar bajo por motivos como falta de comunicación y apoyo afectivo y que refuerzan lo expuesto por Friedemann (1995), en cuanto a la situación de estrés, ansiedad o conflicto que viven las familias con adolescentes, etapa de la vida del ciclo vital humano en la que se encuentran el cien por ciento de estudiantes encuestados.

El hecho de que la dimensión mantenimiento del sistema relacionada con las creencias, hábitos, costumbres, reglas, normas y patrones de crianza haya obtenido la media más alta implica que las familias de los estudiantes mantienen su estabilidad y armonía en relación a estas variables. Sin embargo, el que la dimensión coherencia que engloba las relaciones armónicas, la expresión de lazos de afecto, amor, cariño, comunicación, apoyo y convivencia que le dan a la familia un sentido de unidad, pertenencia y compromiso haya obtenido la media más baja, nos orienta a implementar en los estudiantes intervenciones de fortalecimiento en estas variables, ya que son factores de riesgo para la salud emocional y el desempeño escolar de los estudiantes. Datos que son similares a los de Morales y Rubiano, (2009) en ambas variables y Criado et al (2009) en relación a la dimensión mantenimiento del sistema pero diferentes a la dimensión coherencia.

Lo antes expuesto nos permite concluir que la etapa de vida de los estudiantes (adolescencia), determina de alguna manera la percepción del funcionamiento familiar. 


\section{REFERENCIAS}

1. Consejo Internacional de Enfermeras (CIE, 2002) Cuidar a la familia - las enfermeras dispuestas siempre a ayudarte. Día Internacional de la Enfermera. Carpeta de herramientas de información y acción. Ginebra, Suiza. Recuperado de http://www.icn.ch/es/publications/international-nurses-day/

2. Criado, M. M. L.; Silva, A. N. A.; Torres, D. G. E.; Báez, G. L. J.; Estévez, G. L. V. \& Müller, S. C. (2009). Efectividad de la funcionalidad familiar con padre adolescente en San Gil. Rev. Univ. Ind. Santander. Salud vol.43 no.1 Bucaramanga Apr./Aug.

3. Chávez A. M. L.; Friedemann, M. L. \& Alcorta G. A. (200). Evaluación de la escala de efectividad en el funcionamiento familiar. Desarrollo Científico de Enfermería. México.8 (1):12-8.

4. Friedemann, M. L. (1995). The Framework of Systemic Organization. A Conceptual Approach to Families and Nursing. Sage Publication. EUA: 1-190.

5. Herrera, S. P. M. (1997). La familia funcional y disfuncional, un indicador de salud. Rev. Cub. de Med. Gral. La Habana Cuba. 13(6):591-607.

6. González, Q. N. H. (2004). Funcionalidad familiar en adolescentes de nivel medio y superior. Tesis de Maestría en Ciencias de Enfermería publicada. Facultad de Enfermería. Universidad Autónoma de Monterrey Nuevo León, México. Recuperado de: cdigital.dgb.uanl.mx/te/1020149410.PDF

7. Guadarrama, G. R.; Octavio Márquez, M. O.; Veytia L. M. \& León, H. A. (2011). Funcionamiento familiar en estudiantes de nivel superior Universidad Autónoma del Estado de México. Revista Electrónica de Psicología Iztacala. México. 14, (2), 2011

8. Membrillo, L. A.; Fernández, O. M. A.; Quiroz, P. J. R. \& Rodríguez, L. J. L. (2008). Familia: Introducción al Estudio de sus elementos. Editores de Textos Mexicanos

9. Norma Oficial Mexicana NOM-012-SSA3-2012, que establece los criterios para la ejecución de proyectos de investigación para la salud en seres humanos. Ley General de salud. Diario Oficial de la Federación. México.

10. Morales, M. L. \& Rubiano, Y. (2009). Efectividad de la funcionalidad en las familias con adolescentes de primer semestre de la Fundación Universitaria San Gil. Revista Avances en Enfermería. Santander, Colombia. XXVII (2), p.71-81

11. Paz, S. \& Aymat, A. C. (2007). Problemas en el Desempeño Escolar y su relación con el Funcionalismo Familiar en Alumnos de EGB 1. Revista de la Facultad de medicina. México. D. F. Vol. $8-\mathrm{N}_{\mathrm{o}} .1$

12. Santana, E. I. (2008). Relación entre comunicación intra e inter familiar y la manifestación de permisividad y autoritarismo educativo. Memorias del 6to Congreso Internacional de salud del Adolescente. Instituto Pedagógico Latinoamericano y Caribeño Recuperado de: ttp://www.escuelaspromotorassaludcuba.com/Memorias/pdf

13. Secretaría de Salud (S. S., 2010). Programa de Salud Específico 2007 - 2012. Escuela y Salud. Subsecretaría de Prevención y Promoción a la Salud. México, D. F. 
14. Torres, V. L. E. \& Rodríguez, S. N. Y. (2006). (2006). Rendimiento académico y contexto familiar en estudiantes universitarios. Enseñanza e Investigación en Psicología vol. 11, num. 2: 255-270 juliodiciembre. Recuperado de: redalyc.org Red de Revistas Científicas de América Latina, el Caribe, España y Portugal Sistema de Información Científica. 